Method This study was conducted using the national cardiac arrest registry of OHCA patients with presumed cardiac aetiology who survived to hospital admission from 2009 to 2016. The primary exposure was TTM. The histories of HTN were obtained from patients' medical records. The endpoint was cerebral performance category (CPC) 1 and 2 at discharge and survival to discharge. We compared outcomes between TTM and non-TTM groups using multivariable logistic regression with an interaction term between TTM and HTN for calculating adjusted odd ratios (AORs) and 95\% confidence intervals (CIs) after adjusting for confounding factors.

Results Among 25985 patients following OHCA survived hospital admission with presumed cardiac aetiology. TTM was performed in 12.2\%. TTM group showed better outcomes than non-MTH group: $28.1 \%$ vs $15.5 \%$ for good neurologic recovery $(\mathrm{p}<0.0001)$. AOR $(95 \% \mathrm{CI})$ of TTM for good neurological recovery for all study groups was 1.65 (1.47-1.85). In interaction model (generalised linear mixed model), AOR (95\% CI) of TTM for good neurological recovery was 1.87 $(1.26-2.76)$ in patients without HTN vs $0.87(0.75-1.02)$ in patients with HTN.

Conclusion TTM is associated with good neurological recovery in non-hypertension group, but not significant effect in hypertension group in Korea.

Conflict of interest None

Funding This study was supported by the National Emergency Management Agency of Korea and the Korea Centres of Disease Control and Prevention. The study was funded by the Korea Centres for Disease Control and Prevention.

\section{IMPLEMENTATION OF A BUNDLE OF UTSTEIN TEN STEP RECOMMENDATIONS FROM THE GLOBAL RESUSCITATION ALLIANCE TO IMPROVE SURVIVAL OUTCOMES AFTER OUT-OF-HOSPITAL CARDIAC ARREST IN A METROPOLIS: A BEFORE AND AFTER STUDY}

${ }^{1}$ Jeong Ho Park*, 'Sang Do Shin, ${ }^{1}$ Kyung Jun Song, ${ }^{1}$ Young Sun Ro, ${ }^{1}$ So Yeon Kong, ${ }^{2} \mathrm{Ki}$ Jeong Hong, ${ }^{3}$ Eui Jung Lee. ' Seoul National University Hospital, Korea; ${ }^{2}$ Seoul National University Boramae Medical Centre, Seoul, Korea; ${ }^{3}$ Korea University Anam Hoapital, Seoul Korea

\subsection{6/10.1136/bmjopen-2018-EMS.34}

Aim We aimed to evaluate whether implementation of a bundle of three programs of Utstein ten-step implementation strategy (UTIS) proposed by the Global Resuscitation Alliance improved outcomes after OHCAs.

Method This study was a before-and-after study. All emergency medical services (EMS)-treated adults OHCAs with cardiac aetiology were included. Seoul implemented a bundle of three CPR programs among UTIS programs in 2015;

1. Telephone-CPR (T-CPR) program: a monthly-based and individual dispatcher-targeted quality assurance protocol on telephone-CPR for every OHCA,

2. Rapid CPR program: a multi-tier response, and

3. Feedback CPR program: professional recording of EMS-CPR and feedback to individual team by medical director using high technology defibrillator devices.

The EMS process and outcomes of OHCAs in the study period (2015-2016) were compared with control period (2013-2014). The primary outcome was a good neurological recovery (cerebral performance category 1 or 2). A mixed-effects logistic regression model including random intercepts for district EMS agency level was used to estimate the association between study period with outcomes, adjusted for potential confounders.

Results Total 5968 and 6232 patients were included in the control and study period. T-CPR rate, Rapid CPR, and Feedback CPR in control versus study period were $48.1 \%$ versus $54.2 \%, 1.0 \%$ versus $35.8 \%$, and $27.8 \%$ versus $63.8 \%$, respectively (all p-values $<0.001$ ). Good neurological recovery rate was increased from $5.6 \%$ to $6.5 \%$. In multivariable analyses, the adjusted OR of study period for good neurological recovery was 1.31 (95\% CI: 1.11 to 1.55$)$.

Conclusion The citywide implementation of a bundle of UTIS programs was significantly associated with better OHCA outcomes.

Conflict of interest None

Funding None

\section{NO GENDER DIFFERENCES FOUND IN BYSTANDER CPR IN PRAGUE}

K Vesela*, O Franek. EMS Prague

\subsection{6/10.1136/bmjopen-2018-EMS.35}

Aim The aim of our study is to compare bystander resuscitation ratios between male and female cardiac arrest patients.

Method This is a retrospective analysis of Prague pre-hospital cardiac arrest Utstein-style registry from 2012 to 2016. All patients resuscitated by EMS crew with exception of EMS-witnessed cardiac arrests were included.

Results Total 2302 patients were included. There were 1715 men (M group) and 587 in women (W group) in the study. Bystander CPR was provided in 1368 (79\%) cases in M group and in 477 (81\%) cases in W group. The difference between $M$ and $G$ groups is non-significant $(p=0.31)$.

Conclusion We found no gender differences in the chance of cardiac arrest victim to receive bystander CPR. This finding is in contradiction with the results published by A. Blewer at the American Heart Association's Scientific Sessions in November 2017, where men are more likely to receive CPR in public than women. One possible explanation is the systematic approach of the EMS Prague dispatchers to provide the dispatcher-assisted resuscitation (D-CPR), which can break potential barriers between bystander and cardiac arrest victim.

Conflict of interest None

Funding None

\section{PRE-HOSPITAL RESUSCITATION OF CHILDREN IN THE CITY OF PRAGUE}

K Vesela*, O Franek. EMS Prague

\subsection{6/10.1136/bmjopen-2018-EMS.36}

Aim While there is a lot of studies dealing with out out-ofhospital cardiac arrest (OHCA) in adults, data about OHCA in children age (CH-OHCA) are rarely published. The aim of this study is to describe epidemiology and results of $\mathrm{CH}$ OHCA in City of Prague.

Method This is a retrospective analysis of epidemiology and survival rates of $\mathrm{CH}-\mathrm{OHCA}$ during period from 2003 to 2015. The data are taken from Prague OHCA Utstein-style database. 
Results In the study period, there were 6626 OHCAs attended by Prague EMS, of which 121 met the criteria of $\mathrm{CH}-\mathrm{OHCA}$ (1.8\%, $0.78 \mathrm{CH}-\mathrm{OHCA}$ per 100.000 inhabitants and year). The majority of $\mathrm{CH}-\mathrm{OHCA}$ occurred in age groups $0-0.99(35 ; 28.9 \% ; \mathrm{p}<0.05)$ and $1-1.99(26 ; 21.5 \% \mathrm{p}<0,05)$. In no other age group the number of $\mathrm{CH}-\mathrm{OHCA}$ exceeded 9 (n/s). No CH-OHCA was witnessed by ambulance personnel. Layperson CPR was performed in 95 cases $(78,5 \%)$. The first captured rhythm was most often asystole $(96 ; 79.4 \%)$. Ventricular fibrillation (VF-first subgroup) as the first rhythm was present in 12 patients (10.0\%). The most common cause of $\mathrm{CH}-\mathrm{OHCA}$ was recorded as unknown (39\%). The overall survival rate from $\mathrm{CH}-\mathrm{OHCA}$ (with $\mathrm{CPC} 1-2$ ) was $10.7 \%$, in VF-first subgroup it was $41.7 \%$.

Conclusion $\mathrm{CH}-\mathrm{OHCA}$ is relatively rare event. The most vulnerable group is children under 2 years of age. Small children are most frequently found in asystole, while VF occurs more frequently in age over 15. Even though $\mathrm{CH}-\mathrm{OHCA}$ is uncommon in pre-hospital setting, everyone in the emergency service must be adequately trained for it.

Conflict of interest None

Funding None

\section{A CENTRALISED INTER-FACILITY PATIENT TRANSFER SERVICE IN SOUTHERN FINLAND -THE HELSINKI EXPERIENCE}

${ }^{1} \mathrm{~F}$ Stenius, ${ }^{2} \mathrm{P}$ Holmström, ${ }^{2} \mathrm{~J}$ Etelä, ${ }^{2} \mathrm{~K}$ Sarvikivi, ${ }^{1,2} \mathrm{~T}$ Puolakka*. ${ }^{1}$ Department of Anaesthesiology and Intensive Care Medicine, University of Helsinki; ${ }^{2}$ Emergency Medicine and Services, Helsinki University Hospital, Helsinki, Finland

\subsection{6/10.1136/bmjopen-2018-EMS.37}

Aim Inter-facility patient transfers form an important share of out-of-hospital patient transport. In many countries these transfers are commonly done by small ambulance companies and by the local emergency medical services. The aim of this observational study was to report the operation of the only centralised inter-facility transfer service in Finland.

Method Helsinki University Hospital patient transfer service is responsible of all inter-facility transfers in the region with 1.6 million inhabitants. During a three-month cohort (1.931.11.2016) all patients transferred by the transfer service ambulances were selected for the study. The patient reports were collected and quality deviations during transport were registered based on predetermined criteria.

Results In a total of 3034 transfers, lights and sirens were required in $73(2.4 \%)$ cases. In $45(1.5 \%)$ transfers the patient was accompanied by a physician and in 35 (1.1\%) a nurse from the referring hospital. The most common issues requiring treatment during transport were pain (numeric rating scale $\geq 4)$ in $191(6.3 \%)$ and hypoxemia in 119 (3.9\%) transfers. Moreover, 147 (4.8\%) patients carried microbes important for hospital hygiene. Quality deviations were registered in 338 transfers $(8.4 \%)$. The most common deviations were insufficient or missing written reporting in 244/338 cases (72.2\%). Problems were also encountered with patient monitoring and treatment in 80/338 transfers (23.7\%). Adverse events were registered during 16 transfers $(0.5 \%)$.

Conclusion In daily inter-facility transfer service operations, the need for lights and sirens was uncommon. Acute pain and hypoxemia were the most common issues to be addressed during transport. Adverse events were rare.

Conflict of interest None
Funding None

\section{HEART RATE VARIABILITY AND PRE-HOSPITAL RISK STRATIFICATION OF CHEST PAIN PATIENTS}

${ }^{1}$ Chu En Ting ${ }^{*},{ }^{2,3}$ Nan Liu, ${ }^{4}$ Zhi Xiong Koh, ${ }^{4}$ Dagang Guo, ${ }^{4}$ Janson Cheng Ji NG, ${ }^{4}$ Swee Han Lim, ${ }^{4,5}$ Marcus Eng Hock Ong. 'Duke-NUS Medical School, Singapore; ${ }^{2}$ Health Services Research Centre, Singapore Health Senvices, Singapore; ${ }^{3}$ Centre of Quantitative Medicine, Duke-NUS Medical School, Singapore; ${ }^{4}$ Department of Emergency Medicine, Singapore General Hospital, Singapore; ${ }^{5}$ Health Services and Systems Research, Duke-NUS Medical School, Singapore

\subsection{6/10.1136/bmjopen-2018-EMS.38}

Aim Assessment of chest pain patients often requires extensive investigations, while existing risk-scores are designed for use in Emergency Departments (ED). We aim to develop a risk score for chest pain patients in pre-hospital settings and ambulances by utilising heart rate variability (HRV), a rapid, noninvasive reflection of cardiac stress states.

Method This study analysed 902 chest pain cases presenting to Singapore General Hospital (SGH) ED between 2010 and 2015. The data was divided into a model derivation set (616 cases, $32 \%$ meeting outcomes) and a validation set (286 cases, $28.7 \%$ meeting outcomes). HRV Prediction Model (HRV-PM) for 30 day Major Adverse Cardiac Events (MACE) was derived using backwardstepwise logistic regression. HEART, TIMI and GRACE scores were used as comparators for the HRV-PM.

Results In the derivation set, $66.9 \%$ of patients were male, with a mean (SD) age of 60.71 (12.97). HRV-PM encompasses 9 parameters: $6 \mathrm{HRV}$ variables (triangular interpolation of normal-to-normal intervals, total power, very-low/low/highfrequency power, approximate entropy), and 3 ECG variables (ST-elevation, ST-depression, Q-wave). Validation of HRV-PM shows similar performance to HEART for 30 day MACE prediction with Area Under Receiver-Operating-Curve (AUC) of 0.737 (95\% CI: 0.673 to 0.800$)$ versus 0.739 (95\% CI: 0.679 to 0.799$)$. The addition of 1 set of troponin results in HRV-PM improves the AUC to 0.749 (95\% CI: 0.686 to 0.813), thereby outperforming HEART.

Conclusion HRV-PM is a simple, non-invasive test demonstrating comparable performance with HEART without need of blood-tests. We hope to validate and apply HRV-PM for assessment of chest pain patients by EMS in pre-hospital settings including ambulances.

Conflict of interest None

Funding None

\section{BYSTANDER CPR IN OUT-OF-HOSPITAL CARDIAC ARREST IN SINGAPORE: AGE AND OTHER PREDICTIVE FACTORS}

${ }^{1}$ Jasmine Lim $Y^{*}$, ${ }^{2}$ Win Wah, ${ }^{2}$ Nur Shahidah, ${ }^{2}$ Susan Yap, ${ }^{2}$ Pek Pin Pin, ${ }^{3}$ Yih Yng Ng, ${ }^{4}$ Benjamin SH Leong, ${ }^{5}$ Han Nee Gan, ${ }^{6}$ Desmond R Mao, ${ }^{7}$ Michael YC Chia, ${ }^{8}$ Si Oon Cheah, ${ }^{9}$ Lai Peng Tham, ${ }^{2,10}$ Marcus EH Ong. 'Duke-NUS Medical School, Singapore; ' 2 Department of Emergency Medicine, Singapore General Hospital, Singapore; ${ }^{3}$ Medical Department, Singapore Civil Defence Force, Singapore; ${ }^{4}$ Emergency Medicine Department, National University Hospital, Singapore; ${ }^{5}$ Accident and Emergency, Changi General Hospital, Singapore; ${ }^{6}$ Department of Acute and Emergency Care, Khoo Teck Puat Hospital, Singapore; ${ }^{7}$ Emergency Department, Tan Tock Seng Hospital, Singapore; ${ }^{8}$ Emergency Medicine Department, Ng Teng Fong General Hospital, Singapore; ${ }^{9}$ Children's Emergency, KK Women's and Children's Hospital, Singapore; ${ }^{10}$ Health Services and Systems Research, Duke-NUS Medical School, Singapore

10.1136/10.1136/bmjopen-2018-EMS.39 